\title{
Für eine Verunsicherung des geographischen Blicks: Bemerkungen aus dem Zwischen-Raum
}

Julia Lossau, Bonn

\section{Der Lattenzaun}

Es war einmal ein Lattenzaun, mit Zwischenraum, hindurchzuschaun.

Ein Architekt, der dieses sah, stand eines Abends plötzlich da --

und nahm den Zwischenraum heraus und baute draus ein großes Haus.

Der Zaun indessen stand ganz dumm, mit Latten ohne was herum,

ein Anblick gräßlich und gemein, drum zog ihn der Senat auch ein.

Der Architekt jedoch entfloh

nach Afri -- od -- Ameriko.

(Christian Morgenstern)

\section{Einleitung}

Die Ereignisse im Galgenlied von Christian MorGENSTERN verweisen auf die Irritationen, die auftreten können, wenn die Aufmerksamkeit auf etwas gelenkt wird, was «normalerweise» nicht weiter reflektiert wird, sondern schlicht und einfach (da) ist - und wenn es sich dabei um den Zwischenraum eines Lattenzauns handelt. Irritationen dieser Art ist auch der vorliegende Beitrag gewidmet. Denn im folgenden soll irritiert und verunsichert werden, sollen sicher geglaubte Strukturen hinterfragt und soll das, was auf einen ersten - den geographischen - Blick «ganz alltäglich» und gewohnt erscheint, als bemerkenswert ausgewiesen werden. Daran knüpft sich die Vorgabe, die «Ordnung der Dinge» nicht auf den Punkt oder besser: auf den Begriff zu bringen, sondern aufzuzeigen, daß alles auch ganz anders sein könnte.

Wenn dies die Vorgabe ist, dann liegt es auf der Hand, daß die Ausführungen geradewegs in ein Dilemma führen. Denn dann muß einerseits verunsichert werden; d. h. es darf nicht auf den Punkt gebracht, nicht identifiziert und nicht fixiert werden. Andererseits aber besteht die Notwendigkeit, genau das zu tun - und sei es nur, um die Ausführungen verständlich und nachvollziehbar zu gestalten. In diesem Dilemma deutet sich bereits das erste Anzeichen eines Zwischen-
Raums an - eines Zwischen-Raums, der sich auftut in der Kluft zwischen Kontingenz (d. h. der Notwendigkeit des «Anders-Sein-Könnens») einerseits und der Bewegung eines Abschlusses andererseits.

Dieses Dilemma ist es denn auch, das den folgenden Ausführungen zugrunde liegt. Es kann als charakteristisch für das Denken des Postkolonialismus gelten. Dabei handelt es sich um einen ursprünglich literaturwissenschaftlich geprägten Ansatz, der sich wiederum im Spannungsfeld von postmodernen/ poststrukturalistischen Perspektiven einerseits und marxistischen Perspektiven andererseits bewegt. Schon diese kurze Charakterisierung läßt ahnen, daß das postkoloniale Denken als widerspenstig bezeichnet werden kann. Und in der Tat: Der Postkolonialismus liegt quer zu den konventionellen Disziplingrenzen und vereint unter seiner Überschrift eine große Zahl unterschiedlicher Perspektiven. Diese Zahl ist so groß, daß es angemessen scheint, den Singular bereits in der Einleitung zu streichen und im folgenden nur noch von den Postkolonialismen zu sprechen.

Was die Vertreterinnen und Vertreter der Postkolonialismen bei aller Differenz allerdings zu einen scheint, ist ihre Sensibilität für Fragen der Geographie. Doch während die postkoloniale Kritik ein ganz entschiedenes Interesse am Denken in (Zwischen-)Räumen zeigt, hat sich zumindest die deutschsprachige fachwissenschaftliche Geographie bislang noch kaum mit den entsprechenden Interventionen auseinandergesetzt. Das ist insofern bedauerlich, als die postkoloniale Position des Dazwischen einiges dazu beitragen kann, den geographischen Blick auf die Welt und auf die Ordnung der Dinge in der Welt zu schärfen. Jenen Blick also, der - frei nach dem Diktum von Nicholas SPYKMAN: «For geography does not argue. It just is» (SPYKMAN 1938: 236) - sieht, was in der Welt gegeben ist; allerdings ohne dabei zu sehen, daß alles auch ganz anders sein könnte. Und so soll im folgenden aufgezeigt werden, inwieweit das widersprüchliche Denken im postkolonialen Zwischen-Raum dazu beitragen kann, den geographischen Blick zu bereichern.

$\mathrm{Zu}$ diesem Zweck soll zunächst der postkoloniale Ansatz oder auch: die postkoloniale Brille vorgestellt werden. Während das Pferd damit gewissermaßen von hinten aufgezäumt wird, wird es im dritten Teil darum gehen, mit dieser Brille auf die Geographie zu schauen. Dabei läßt bereits die Überschrift «Welt-Ordnung vom Klassenzimmer bis zum Kanzleramt: Der geographische Blick» ahnen, daß nicht bloß die fach- 
wissenschaftliche Geographie angesprochen ist. Vielmehr muß gleichermaßen auf den populären geographischen Blick eingegangen werden. Diese «doppelte Sicht» ergibt sich aus der Einsicht, daß das in einer bestimmten Disziplin verhandelte Wissen nicht auf diese Disziplin beschränkt ist, sondern vom Klassenzimmer bis zum Kanzleramt auf unzähligen Feldern zum Tragen kommt (vgl. hierzu auch GrEGORY 1994). Im vierten Teil wird das Konzept «Verunsicherungen», das sich aus dem Denken im ZwischenRaum ergibt, unter der Überschrift «Entdecken Sie Kiloland!» in die Tat umgesetzt. Dabei wird es allerdings nicht allein um die (dekonstruktive) Verunsicherung des derzeit wohl wirkungsmächtigsten Welt-Bilds gehen. Vielmehr soll - im Einklang mit den dekonstruktiv-rekonstruktiven Bewegungen der Postkolonialismen und in direkter Auseinandersetzung mit dem zu dekonstruierenden Welt-Bild - eine Praxis skizziert werden, die es erlaubt, andere und wenn möglich bessere Welt-Bilder zu skizzieren.

\section{Ein Denken im Zwischen-Raum}

Soll das Denken im Zwischen-Raum genauer betrachtet werden, so bietet es sich an, dasjenige Feld zu beleuchten, auf dem diese Zwischen-Position maßgeblich entwickelt wurde. Dabei handelt es sich - neben demjenigen der sog. gender studies - um das widersprüchliche Feld der Postkolonialismen (erkenntnistheoretische Parallelen zwischen gender studies und postkolonialer Kritik zeigt etwa NaSSEHI 1999 auf). Und tatsächlich besteht eine der größten Gemeinsamkeiten inmitten der vielen Differenzen darin, daß die «PostPerspektiven» gegen jenen machtvollen Akt rebellieren, der Heterogenes und Vielfältiges zwangsweise fixiert und auf einen gemeinsamen Nenner bringt. Die Kritik an diesem "Gestus der Begriffsherrschaft» (ButLER 1993:34) rührt insbesondere daher, daß diese Perspektiven vor einem Hintergrund operieren, demzufolge alles ebenso gut ganz anders sein könnte.

Es sind wohl gerade ihre «kontingenten Grundlagen» (BUTLER 1993), aufgrund derer ihren Vertreterinnen und Vertretern immer wieder vorgeworfen wird, sie redeten einem nihilistischen Relativismus das Wort. Demgegenüber soll hier die These vertreten werden, daß eine Absage an die eine, als selbstverständlich geltende Wirklichkeit nicht zwangsläufig impliziert, normative Fragen als "sinnlos» zu verwerfen, sondern daß diese Absage im Gegenteil als Vorbedingung für eine engagierte gesellschaftspolitische Kritik gelten kann (BUTLER 1993: 36). Zur Untermauerung dieser These sei kurz aufgezeigt, womit zu rechnen ist, wenn - wie im Falle eines objektivistischen Wissenschaftsverständnisses - vermeintliche Fakten postuliert werden; wenn also nicht Kontingenz, sondern
Objektivität und Allgemeingültigkeit postuliert bzw. in den Vordergrund gespielt werden. Dann droht zum einen (und eher erkenntnistheoretisch gesprochen) die Theologie-Werdung aller Wissenschaft. Denn dann wird Realität aus einer Perspektive des Nirgendwo gedacht - und damit «aus der Perspektive Gottes (...), dem allein das Privileg zusteht, eine Perspektive des Nirgendwo einzunehmen, und der deshalb ohne Perspektive auskommt» (Nassehi 1999: 354). Zum anderen aber (und nun eher praktisch-politisch gesprochen) werden andere mögliche Wahrheiten und andere mögliche Erzählungen - sozusagen unter der Hand marginalisiert und ausgeschlossen.

Insofern die besagten Perspektiven auf diese beiden Aspekte des universalisierenden Gestus aufmerksam machen, kann von einer nihilistischen Position keine Rede sein, und es wird deutlich, warum eine Absage an die Möglichkeit objektiver Erkenntnis als Vorbedingung für eine politisch-engagierte Kritik betrachtet werden kann. Zwar differiert das Engagement, das dabei an den Tag gelegt wird, von Beitrag zu Beitrag. Aber alle Beiträge zielen darauf $a b$, jenen Wahrheiten und Identitäten zu ihrem Recht zu verhelfen, die sich im «blinden Fleck» universalistischer Erzählungen befinden. Da die postkoloniale Kritik dabei auf die vielfältigen Erfahrungen kolonialer Marginalität abzielt, die auch nach der formalen Entkolonialisierung noch längst nicht der Vergangenheit angehören (vgl. GANDHI 1998, GRIMm 1997), ist sie insbesondere von der De- und Rekonstruktionsarbeit auf dem Feld der kulturellen Differenz geprägt. Verhandelt werden allerdings nicht die «natürlichen», essentialistischen Kategorien der Rasse bzw. der Ethnie, sondern solche, die im Lauf langer Kämpfe um Repräsentation konstruiert worden sind. Damit rekonstruieren die Vertreterinnen und Vertreter der Postkolonialismen partielle, fragmentierte Identitäten jenseits des essentialistischen Gegensatzes vom «Eigenen» und «Anderen» (vgl. HaLl 1994).

Möchte man die bisherigen Ausführungen zusammenfassen, dann wird man nicht anders können, als die genannten Ansätze in einem Dazwischen zu plazieren - oder eben in einen Zwischen-Raum zwischen Dekonstruktion und Rekonstruktion bzw. zwischen Identität und Differenz. Vor diesem Hintergrund ist Leela Gandhi zuzustimmen, wenn sie den Vertreterinnen und Vertretern der Postkolonialismen ein Denken bescheinigt, das sich «zwischen Nationalismus und Internationalismus, strategischem Essentialismus und Hybridität, Solidarität und Zerstreuung, einer Politik der Struktur oder Totalität und einer Politik des Fragments» (GANDH 1998: ix; Üs. d. Verf.) bewegt. Allerdings sollte dieses Denken im Zwischen-Raum nicht dahingehend verstanden werden, als strebe es danach, die Widersprüche zwischen den genannten 
Polen aufzuheben. Vielmehr kann (und sollte) es als ein Denken verstanden werden, das von der Spannung lebt, «unvereinbare Dinge beieinander zu halten, weil beide oder alle notwendig und wahr sind" (HaRAWAY 1995: 33). Und so ungewohnt dieses Aushalten von Widersprüchen auch sein mag, so ist es doch gerade das Denken in Kategorien des Dazwischen, das die Produktivität der «Post-Perspektiven» ausmacht.

\section{Welt-Ordnung vom Klassenzimmer bis zum Kanzleramt: Der geographische Blick}

«What I find myself doing (...) is rethinking geography». Dieses Zitat stammt nicht, wie man vielleicht vermuten könnte, aus dem Kontext der geographischen scientific community. Vielmehr war es der Literaturwissenschaftler EDWARD SAID, der von sich behauptete, die Geographie neu/umzudenken (SAID zitiert in GREGORY 1995b: 447). Und in der Tat können seine Schriften als sehr weitgehender Versuch gelesen werden, die Geographie auf Grundlage der skizzierten ZwischenPosition neu zu verhandeln. Im Zuge dieser Verhandlung hat sich im Lauf der letzten zehn Jahre - insbesondere im angelsächsischen Kontext - auch eine postkoloniale Geographie entwickelt. Obwohl eine wichtige Aufgabe der postkolonialen Geographinnen und Geographen in einer Kritik des disziplinären Diskurses besteht, beschränken sie ihre Interventionen längst nicht auf die Geographie in ihren disziplinären Grenzen. Im Vordergrund steht vielmehr die Auseinandersetzung mit den Welt-Bildern des populären geographischen Diskurses (vgl. Gregory 1994: 11), die - vom Klassenzimmer bis zum Kanzleramt - in unzählige Topographien von Macht und Wissen eingelassen sind (vgl. auch LosSAU 2000).

Als Klassiker in bezug auf diese populären Geographien gilt SAIDs Buch «Orientalism» (SAID 1978), in dem er - in Anlehnung an Michel Foucaults Untersuchungen zum Diskursbegriff und insbesondere dessen «Archäologie des Wissens» (Foucault 1977, 1981) aufzeigt, wie der Orient in den entsprechenden Diskursen des französischen und britischen Imperialismus als das «Andere» Europas produziert wurde. Auch in seinen späteren Arbeiten unterstreicht SAID den zentralen Stellenwert, den geographische Macht/WissenSysteme oder imaginative Geographien innerhalb der imperialen Diskurse innehaben: Der Imperialismus stelle eine Form geographischer Gewalt dar, mit deren Hilfe die ganze Welt erforscht, kartiert und letztlich unter (westliche) Kontrolle gebracht werde (SAID 1994). Als entscheidendes Moment dieser Gewalt gilt die Fixierung und Normalisierung der vermeintlich homogenen Kategorien des «Eigenen» und der "Anderen» - handelt es sich bei den imaginativen Geographien doch um «spannungsgeladene Konstel- lationen von Macht, Wissen und Räumlichkeit, die in einem 'Hier zentriert sind und auf ein 〈Dort> projiziert werden» (GREGORY 1995a: 29; Üs. d. Verf.).

In dieser Konzeption von «Geographie» wird der populär-geographische Blick - der Blick auf vermeintlich natürliche imaginative Geographien also - seines Status als Prophet einer gleichsam naturgegebenen Wahrheit beraubt. Er wird umgekehrt als eine diskursive Praxis gefaßt, mit deren Hilfe die scheinbar natürliche geographische Wirklichkeit erst produziert wird. Denn in dieser Konzeption wird deutlich, daß die diskursive (Re-)Produktion einer bestimmten Wirklichkeit immer auch eine Verortung der Objekte und Identitäten beinhaltet, die wiederum die «Ordnung der Dinge» natürlich erscheinen läßt - als müsse sie so und könne sie nicht auch ganz anders sein.

Inwiefern aber kann die Logik dieses Blicks auch als charakteristisch für den disziplinären geographischen Diskurs gelten? Zur Beantwortung dieser Frage bietet es sich an, den Ursprüngen dieser Logik auf die Spur zu kommen. Dabei kann mit Foucault (1974) argumentiert werden, daß sich während des 17. Jahrhunderts ein neues Erkenntnisprinzip etablierte, das sich, um einen Begriff von ARMIN NASSEHI (1999) «zweckzuentfremden», durch eine «Paradoxie der Sichtbarkeit» auszeichnete. Was aber bedeutet das Paradoxie der Sichtbarkeit?

Ohne ins Detail zu gehen, bedeutet es, daß sich zu dieser Zeit insbesondere innerhalb des naturgeschichtlichen Felds eine nicht anders als naiv zu bezeichnende «Benennung des Sichtbaren» (Foucault 1974:173) vollzog: Das, was gesehen wurde, wurde durch das, was gesagt wurde, auf eine ganz bestimmte Weise sichtbar gemacht, auf daß es so und nicht anders gesehen wurde. Denn die Naturgeschichte hatte, wie FouCault schreibt, die Aufgabe, «die Sprache dem Blick sehr nahe zu bringen und die betrachteten Dinge möglichst in die Nähe der Wörter zu rücken» (Foucault 1974: 173).

Genau dies ist es nun, was als Paradox der Sichtbarkeit bezeichnet werden kann: der naive Glaube an die Natürlichkeit evidenter Dinge, die - in eine ganz bestimmte Ordnung gebracht - sichtbar gemacht wurden und folglich so und nicht anders gesehen wurden. Und genau dies ist es auch, was sich innerhalb der zweiten Hälfte des 18. Jahrhunderts im Rahmen des «frühen» geographischen Diskurses vollzog und letztlich zur Entstehung der modernen geographischen Wissenschaft führen sollte (vgl. zum folgenden GreGORY 1994: 15ff.; STODDART 1986: 32ff.): Es manifestierte sich jener Blick, der die vermeintlich natürliche Natur mittels Beschreibung, Klassifikation und Vergleich vor sich ausbreitete und so eine panoptische Position konstruierte, von der aus geordnet und objektiviert werden konnte. Dieser naturalisierende Blick aber richtete sich nicht nur auf «Natur», sondern auch auf Völker und Gesellschaften (STODDART 1986: 
32ff.) - und erfuhr damit genau jene "Ausweitung», die die Formierung des klassischen geographischen Gegenstands der «Raumgestalten» möglich machte, «in denen «Natur», 〈Kultur〉 und 〈Gesellschaft〉 zu einer Einheit zusammengewachsen» (WERLEN 1997:44) sind. Man kann sich die Logik dieses Blicks - anschaulich vereinfacht - auch folgendermaßen vorstellen:

«Wenn Jemand ein Ding hinter einem Busche versteckt, es eben dort wieder sucht und auch findet, so ist an diesem Suchen und Finden nicht viel zu rühmen: so aber steht es mit dem Suchen und Finden der (Wahrheit innerhalb des Vernunft-Bezirkes. Wenn ich die Definition des Säugethieres mache und dann erkläre, nach Besichtigung eines Kameels: Siehe, ein Säugethier, so wird damit eine Wahrheit zwar ans Licht gebracht, aber sie ist von begrenztem Werthe, ich meine, sie ist durch und durch anthropomorphisch und enthält keinen einzigen Punct, der <wahr an sich>, wirklich und allgemeingültig, abgesehen von dem Menschen, wäre» (NIETZSCHE 1988: 883).

Damit aber kann festgehalten werden, daß es sich bei den vermeintlich natürlichen Landschaften, Ländern oder «Kulturräumen», die (zusammen mit den «darin lebenden Menschen») die Welt der traditionellen Geographie bildeten, um Räume «konstruierter Sichtbarkeit» (RAJCHMAN 1991 zit. in GREGORY 1998: 23; Üs. d. Verf.) handelte, die durch den spezifisch geographischen Blick erst «erfunden» wurden. Und wenngleich hier nicht behauptet sei, es gebe in der fachwissenschaftlichen Geographie bis heute nur diesen einen naturalisierenden Blick auf evidente Gegenstände, so hat es doch oftmals den Anschein, als sei die «Grundeinstellung» erhalten geblieben. Diese Grundeinstellung besteht in einer objektivistischen Sicht der Dinge, in der eine sichtbar gemachte Wirklichkeit als die einzig mögliche Wirklichkeit angesehen wird.

Spätestens an dieser Stelle zeigt sich, warum die oben eingeführte Unterscheidung zwischen einem populären Diskurs einerseits und einem disziplinären Diskurs andererseits lediglich aus heuristischen Gründen getroffen werden kann: weil es aus einer Zwischen-Position heraus betrachtet letztlich keine Rolle spielt, ob imaginative Geographien nun innerhalb oder außerhalb der fachwissenschaftlichen Geographie (re-) produziert werden. Die Verortung essentialistischer Entitäten auf vermeintlich natürlicher Grundlage - sei sie nun geographischer, (sicherheits-)politischer,(politik-)wissenschaftlicher oder auch "ganz alltäglicher» Art - muß vielmehr immer dazu führen, daß die Kontingenz der Wirklichkeit auf überschaubare geographische Abstraktionen reduziert und in eine ganz bestimmte Ordnung gebracht wird. Und zwar in eine Ordnung, die andere Ordnungen ausschließen und andere Wahrheiten marginalisieren muß.

Solche Welt-Ordnungen gilt es aus der Zwischen-Position heraus zu dekonstruieren; d. h. es gilt aufzuzeigen, daß die geographische Wirklichkeit nicht per se ist, sondern immer wieder aufs neue erst (re-)produziert wird. Aber nicht nur das: Mit der Dekonstruktion verbindet sich immer auch der Versuch, andere und wenn möglich bessere Welt-Bilder zu skizzieren.

\section{Entdecken Sie Kiloland! - De- und Rekonstruktion eines Welt-Bilds}

Im Frühjahr 2000 berichtete die «Süddeutsche Zeitung», daß es auf der Insel Kiloland vor der afrikanischen Westküste zu gewalttätigen Auseinandersetzungen zwischen der Mehrheit der Adonia und der Minderheit der Butonia gekommen sei (vgl. PoDaK 2000). Angesichts der bürgerkriegsähnlichen Zustände, so der Bericht weiter, habe die Regierung Kilolands die Vereinten Nationen zu Hilfe gerufen. Die Vereinten Nationen wiederum hätten die Entsendung einer internationalen Friedenstruppe beschlossen und NATO und WEU ein entsprechendes Mandat erteilt. Aber da die NATO zu diesem Zeitpunkt schon anderweitig engagiert gewesen sei, habe die WEU nach Absprachen mit der Allianz beschlossen, den Frieden auf Kiloland unter Rückgriff auf NATO-Mittel im Alleingang wiederherzustellen. Diesem Alleingang der europäischen Militärorganisation war es wohl auch geschuldet, daß sich die Medien weniger mit der Entdeckung Kilolands als vielmehr mit den infrastrukturellen Details des WEU-Einsatzes beschäftigten. Vor dem Hintergrund dieser Details aber geriet das kaum entdeckte Kiloland wieder in Vergessenheit, und auch das Schicksal einer kleinen Gruppe europäischer Zivilisten, die Gefahr gelaufen waren, zwischen die Fronten zu geraten, schien bis auf den heutigen Tag keine weitere Meldung wert.

Es darf angenommen werden, daß von Kiloland auch in Zukunft nicht mehr allzu oft die Rede sein wird. Denn «natürlich» existiert diese Insel nicht «wirklich». Sie stellt vielmehr eine Konstruktion dar - eine Konstruktion innerhalb einer gemeinsamen «Krisenmanagement-Übung» von NATO und WEU, die im Frühjahr 2000 unter dem Codenamen CMX/Crisex 2000 durch-«gespielt» wurde. Dieses «Krisenmanagement-Spiel» kann als trefflicher Ausdruck des derzeit wohl wirkungsmächtigsten Welt-Bilds gelten - und zwar nicht nur der europäischen Außen- und Sicherheitspolitik. Es repräsentiert die Welt als eine, die von ethnisch-kulturellen Konfliktlinien durchzogen ist. Den entscheidenden Impuls dürfte hier der Politikwissenschaftler SAMUEL Huntington gegeben haben, der die neue Wirklichkeit der internationalen Beziehungen in seinem Aufsatz über den «Clash of Civilizations» (HunTINGTON 1993) öffentlichkeitswirksam auf den Punkt gebracht hat. Nach dem Ende des Kalten Krieges, so Huntington, sei diejenige Bruch- 
linie wieder relevant geworden, die bereits um 1500 das westliche vom orthodoxen Christentum sowie dem Islam getrennt habe. Damit habe der «Samtene Vorhang der Kultur» den «Eisernen Vorhang der Ideologie» ersetzt, der allerdings so samten gar nicht sei, sondern zuweilen eine blutige Konfliktlinie darstelle (HunTINGTON 1993: 31).

Die Argumentation Huntingtons kann als idealtypisch für die oben skizzierte Logik des geographischen Blicks gelten. Dabei sei an dieser Stelle dahingestellt, ob sich der Autor über die Wirkungsmacht seiner Beiträge im Klaren ist oder nicht. Jedenfalls tut er nichts anderes, als ein ethnisch fragmentiertes Welt-Bild zu produzieren, in dessen gedachter Mitte die partikularen Interessen des «Eigenen» stehen. Mit anderen Worten: Er nimmt eine radikale Schließung der kontingenten Wirklichkeit vor und reduziert sie auf die überschaubaren geographischen Imaginationen des «Eigenen» und des «Anderen».

Nun erinnert aber $C M X /$ Crisex 2000 daran, daß geographische Imaginationen keine bunten Blumen auf der semantischen Spielwiese der Politikwissenschaften darstellen. Im Gegenteil: «Wissen ist Macht - geographisches Wissen ist Weltmacht» (zit. etwa in Brogiaro 1998). Noch pointierter hat der französische Geograph Yves LACOSTE den Zusammenhang zwischen Macht und geographischem Wissen formuliert: «La géographie, ça sert, d'abord, à faire la guerre» (LACOSTE 1985). Zwar vermag die Rhetorik von CMX/Crisex 2000 durchaus den Anschein zu vermitteln, als sei «la guerre» mittlerweile durch «das Krisenmanagement» ersetzt worden. Euphemismen dieser Art sollten allerdings niemanden daran hindern, die realen Effekte zu betrachten, die der geographische Blick zeitigt. Eine Betrachtung dieser Realität des Diskurses aber verführt förmlich dazu, der Dekonstruktion des in ethnisch homogene Blöcke zerfallenen Welt-Bilds eine Bewegung der Rekonstruktion folgen zu lassen. Und so soll im folgenden versucht werden, dem konflikthaften Weltbild eines Samuel Huntington, in dessen Mittelpunkt das «Eigene» ruht, ein anderes und wenn möglich besseres Bild der Welt entgegenzusetzen.

Zu diesem Zweck bietet es sich an, einmal zu prüfen, inwieweit von den Antagonistinnen und Antagonisten Huntingtons ein solch anderes Bild gezeichnet wird. Zwar können die vielen Kritiken des Clash of Civilizations kaum über einen Kamm geschoren werden reichen die Erwartungen doch von der Hoffnung auf einen verstärkten interkulturellen Austausch über die tendenzielle bis hin zur absoluten Auflösung kultureller Unterschiede im «globalen Dorf» der Weltgesellschaft. Was allerdings viele, die dem «Kampf der Kulturen» ablehnend gegenüberstehen, eint, ist der Bezug auf einen Begriff, dessen aktuelle Popularität ihresgleichen sucht: den Begriff der Globalisierung. Und vielleicht kann der hoffnungsvolle Tenor als symptomatisch für diejenigen Positionen gelten, die im Rahmen der sozialwissenschaftlichen und soziologischen Debatten zum Tragen kommen (vgl. hierzu NASSEHI 1998: 151ff.): Dort scheint unter dem Stichwort «Globalisierung» weniger der Kampf als vielmehr der Ausgleich zwischen den Kulturen antizipiert zu werden. Mit anderen Worten: Die sozialwissenschaftlichen Beiträge sind vielfach durch die Hoffnung gekennzeichnet, daß die Welt zu derjenigen «Einen Welt» zusammenwächst, deren Bild schon in den 70er Jahren von Ökologie- und «Dritte-Welt»-Bewegungen gezeichnet wurde - wenn auch als Provokation.

Aber so «schön» das Bild dieser «Einen Welt» auch sein mag: Es stellt sich die Frage, ob diejenigen, die HunTINGTON auf diese Art und Weise entgegentreten, letztlich über dessen Thesen hinauskommen können. Damit sei - um möglichen Mißverständnissen vorzubeugen - keinesfalls angedeutet, seine Kritikerinnen und Kritiker argumentierten nicht «besser» im Sinne einer universalistischen Moral. Im Gegenteil: Ihre Einwände beruhen vielfach auf den Grundsätzen einer universalistischen Liberalität, die eine an partikularistischen Interessen orientierte Machtpolitik ausschließen und ihr die wohlwollende Achtung «anderer» ethnischer Traditionen entgegensetzen möchte - ganz im Sinne der Gleichheit und Freiheit aller Menschen.

Wenn hier also bezweifelt wird, daß die Kritikerinnen und Kritiker Huntingtons über die Thesen des von ihnen Kritisierten hinauskommen können, dann nicht, weil ihnen eine Verfehlung humanistischer Ansprüche unterstellt würde. Was ihnen hingegen unterstellt wird, ist folgendes: Obwohl - oder, besser noch, gerade weil - sie vor dem Hintergrund einer universalistischhumanistischen Folie argumentieren, können sie nicht über die Rede von konflikthaften kulturellen Differenzen hinauskommen. Und zwar deshalb, weil auch sie letztlich nichts anderes tun, als essentialistische (National-)Kulturen zu verorten, d. h. an ein «eigenes» und «eigentliches» Territorium zu binden. Denn auch sie unterlegen wie selbstverständlich die ontologische Existenz verschiedener Kulturen; auch sie gehen wie selbstverständlich davon aus, daß die jeweilige «Kultur», die jeweilige «Zivilisation» oder auch die jeweilige «Gesellschaft» eine ontologische Einheit darstellt: einen identitätsstiftenden, durch gesellschaftliche Gemeinschaft harmonisierten Zusammenhang (vgl. NASSEHI 1997: 189ff.). Es dürfte auf der Hand liegen, daß Konzepte, die diesen Mythos gesellschaftlicher Gemeinschaft transportieren, personelle und kollektive Identitäten in ein "stahlharte[s] Gehäuse der Zugehörigkeit» zwängen (NASSEHI 1997). Folglich können sie kaum dazu beitragen, (national-)kulturelle Grenzen zu überwinden. Sie müssen sich im Gegenteil als Bestandteil desjenigen Problems erweisen, das zu lösen sie angetreten sind. 
Dennoch können sie, sozusagen invers und zusammen mit dem in homogene ethnische Blöcke zerfallenen Welt-Bild, dazu beitragen, andere und bessere Welt-Bilder zu skizzieren. Denn im Zwischen-Raum des in ethnisch homogene Blöcke zerfallenen WeltBilds einerseits und des universalistischen, auf harmonischen Ausgleich bedachten Welt-Bilds andererseits kann eine Praxis entwickelt werden, die es erlaubt, immer wieder andere, subversive und auch bessere Momentaufnahmen der «Einen Welt» zu skizzieren. Diese Praxis beansprucht keinen «eigenen» Raum,sondern bewegt sich kontinuierlich zwischen ihren beiden Anderen. Im Rahmen ihrer permanenten Ortswechsel, die einem unablässigen Hin und Her gleichkommen, aber scheinen immer wieder andere, vorläufige WeltBilder auf: Welt-Bilder, die sich oft nur «um Haaresbreite» von ihren jeweils Anderen unterscheiden und die sich dennoch (oder gerade deshalb) allen Versuchen der Fixierung durch einen panoptischen Blick entziehen.

Diese (Vexier-)Bilder verzichten ebenso auf den Anspruch, allgemeingültig oder objektiv zu sein, wie sie ohne die fixierende Verortung eines essentialistischen «Eigenen» und «Anderen» auskommen. Und wenn sie zudem das Adjektiv «besser» für sich in Anspruch nehmen, dann ist damit kein absolutes «Besser» gemeint, wie es etwa mit der universalistischen Moral der Aufklärung verbunden ist. Zwar interveniert die skizzierte Praxis gegen die Durchsetzung partikularistischer «Eigen»-Interessen auf Kosten von «Anderen». Aber sie erinnert auch an den inklusivexklusiven, den falsch universalisierenden Charakter großer Erzählungen im Sinne der conditio humana. Damit macht sie nicht nur darauf aufmerksam, daß der Traum einer universellen Moral als ausgeträumt zu betrachten ist (vgl. PODAK 2000). Vielmehr strebt sie auch danach, jenen Wahrheiten und Identitäten zu ihrem Recht zu verhelfen, die gleichsam «unter der Hand» immer wieder aufs neue ausgeschlossen werden. Dabei kommt sie allerdings nicht umhin, den vorläufigen, partiellen und fiktionalen Charakter der von ihr verhandelten (und letztlich aller) Wahrheiten und Identitäten zu betonen.

Wie wichtig letzteres ist, kann wohl nicht oft genug betont werden - ist es doch gerade das Denken in Differenzen, dem diese Praxis ihre dissidenten Potentiale überhaupt nur verdankt. Und so sei abschließend daran erinnert, daß das Denken im Zwischen-Raum immer darauf abzielen muß, sich zwischen Identität und Differenz zu bewegen, ohne die Unvereinbarkeit dieser beiden Pole aufzuheben. Anders ausgedrückt: So wichtig es auf dem Terrain der Dekonstruktionen ist, den Pol der Identität nicht zu vergessen, so wichtig ist es auf dem Terrain der Rekonstruktionen, den Pol der Differenz nicht aus den Augen zu verlieren. Denn würde die Differenz aus den Augen verloren, dann täte das Denken im Zwischen-Raum letztlich nichts anderes als das, was es dem geographischen Blick zum Vorwurf macht: Es würde die Welt von einem freischwebenden Aussichtspunkt aus ordnen - ohne zu bedenken, daß es einen solchen Aussichtspunkt schon allein deshalb nicht geben kann, weil es die Welt als solche nicht gibt, und daß schlicht nichts gedacht werden kann, was vor seiner Bezeichnung von Interesse wäre.

\section{Für eine Verunsicherung des geographischen Blicks}

Inwiefern also kann das Denken im Zwischen-Raum dazu beitragen, den geographischen Blick zu bereichern? Sein Beitrag liegt gewissermaßen darin, das eben Gesagte aufzuzeigen: daß es einen freischwebenden Aussichtspunkt ebensowenig geben kann wie die eine, natürliche geographische Wirklichkeit. Damit fordert es dazu auf, sich weniger auf die Betrachtung eines Gegenstands als darauf zu konzentrieren, auf welche Art und Weise dieser Gegenstand - auch innerhalb des eigenen Arbeitens - betrachtet und damit (re-)produziert wird (in bezug auf die Soziologie vgl. NASSEH 1999:359). Mit anderen Worten: Es fordert dazu auf, vertraute Ordnungen in Frage zu stellen, felsenfeste Überzeugungen zu überdenken und gewohnte Denkschemata zu hinterfragen.

Ganz im Sinne dieser permanenten Verunsicherung (insbesondere auch in bezug auf das eigene Arbeiten) sei abschließend noch eine Schwierigkeit thematisiert, der sich die postkoloniale Kritik immer wieder aussetzen muß. Dabei handelt es sich um die Schwierigkeit, letztlich nicht über den identifizierenden Gestus der Begriffsherrschaft, in dessen Mittelpunkt das westliche Subjekt steht, hinauskommen zu können. Diese Schwierigkeit muß wohl als «größte anzunehmende Schwierigkeit» betrachtet werden, denn sie stellt - und beantwortet zugleich - die Frage, ob nicht auch die Praxis der Postkolonialismen letztlich «immer noch die 〈Psyche〉 des Westens repräsentiert, den Spiegel, in dem das Subjekt des Westens sich selbst festhält, selbst wenn es dies nur tut, um sein eigenes Verschwinden zu beklagen» (DhAREShwar 1990: 232; Üs. d. Verf.). Wenn aber diese Schwierigkeit nicht von der Hand zu weisen ist, dann stellt sich die Frage nach ihren Konsequenzen in bezug auf die skizzierte Verunsicherungs-Praxis. Denn wenn die postkolonialen Projekte letztlich zum Scheitern verurteilt sind, weil sie trotz aller Bemühungen nichts anderes zu (re-)produzieren vermögen als das, was sie «eigentlich» zu überwinden gehofft hatten - dann stellt sich die Frage, ob überhaupt für Verunsicherung plädiert werden sollte.

Aber läßt diese Schwierigkeit tatsächlich keine andere Wahl als die schlechte Wahl der Resignation? Oder bezeichnet sie nicht vielmehr dasjenige Dilemma, das bereits in der Einleitung thematisiert wurde? Dasjenige Dilemma also, das darin besteht, immer wieder 
aufs neue Vielheiten produzieren und die Grenzen sprachlicher Bezeichnungen überschreiten zu wollen? Und doch immer schon zu wissen, daß Vielheiten nicht produziert und die Grenzen sprachlicher Bezeichnungen nicht überschritten werden können?

Wenn dieses Dilemma aber schon einleitend bestand, und der Beitrag trotzdem geschrieben wurde, dann sollte es auch im Anschlu $\beta$ an den Beitrag nicht dazu führen, entmutigt die Hände in den Schoß zu legen. Mit anderen Worten: Auch wenn (oder gerade weil) die besagte Schwierigkeit ernst genommen werden muß; auch wenn (oder gerade weil) es zu keiner Zeit gelingen konnte, «über den Begriff hinauszugelangen» (ADORNo 1982), so besteht kein Anlaß zur Resignation. Im Gegenteil - hat sich doch im Lauf des Beitrags gezeigt, daß es gar nicht im Sinne eines Denkens im Zwischen-Raum sein kann, über den Begriff hinauszugelangen. Das Ziel muß vielmehr in der «eigentlich» unmöglichen Verweigerung einer Struktur bestehen, «die man angreift und zugleich verinnerlicht hat» (SPIVAK 1993 zit. bei Grimm 1997). Und damit nicht zuletzt den eigenen Blick immer wieder aufs neue zu... verunsichern.

\section{Dank}

Mein Dank gilt Andrea Kofler, Bettina Fredrich und Doris Wastl-Walter, die es mir ermöglicht haben, das Konzept «Verunsicherungen» im Rahmen des Kulturgeographischen Kolloquiums an der Universität Bern (Wintersemester 2000/2001) einer anregenden Diskussion auszusetzen. Danken möchte ich auch Roland Lippuner, der das diesem Beitrag zugrundeliegende Vortragsmanuskript kritisch kommentiert hat, und der Bonner Romanistin Esther Winkelmann, die die Zusammenfassung ins Französische übersetzt hat.

\section{Literatur}

Adorno, T. W. (1982): Negative Dialektik. - 3. Auflage, Frankfurt am Main: Suhrkamp.

Brogiato, H. P. (1998): «Wissen ist Macht - geographisches Wissen ist Weltmacht». Die schulgeographischen Zeitschriften im deutschsprachigen Raum (1880-1945) unter besonderer Berücksichtigung des Geographischen Anzeigers. - = Materialien zur Didaktik der Geographie 18, Trier.

Butler, J. (1993): Kontingente Grundlagen: Der Feminismus und die Frage der «Postmoderne». - In: BENHABIB, S. et al. (Hrsg.): Der Streit um Differenz. Feminismus und Postmoderne in der Gegenwart. - Frankfurt am Main: 31-58.

DHARESHWAR, V. (1990): The predicament of theory. In: Kreiswirth, M. \& M. Cheetham (Hrsg.): Theory between the disciplines: Authority / vision / politics, Ann Arbor: 231-250.

Foucault, M. (1974): Die Ordnung der Dinge. Eine
Archäologie der Humanwissenschaften. Frankfurt am Main: Suhrkamp.

Foucault, M. (1977): Überwachen und Strafen. Frankfurt am Main: Suhrkamp.

Foucault, M. (1981):Archäologie des Wissens. - Frankfurt am Main: Suhrkamp.

GandhI, L. (1998): Postcolonial Theory. A Critical Introduction. - New York: Columbia.

GREGORY, D. (1994): Geographical imaginations. Cambridge, Oxford: Blackwell.

GREGorY, D. (1995a): Between the book and the lamp: imaginative geographies of Egypt, 1849-50. - In: Transactions of the Institute of British Geographers 20 (NS): 29-57.

GrEgoRy, D. (1995b): Imaginative geographies. - In: Progress in Human Geography 19: 447-485.

GREGORY, D. (1998): Power, knowledge and geography. - In: Gebhardt, H. \& P. Meusburger (Hrsg.): Explorations in Critical Human Geography. Hettner-Lecture 1997 with Derek Gregory. - = Hettner-Lectures 1, Heidelberg: 9-40.

Grimm, S. (1997): Postkoloniale Kritik. Edward Said, Gayatri C. Spivak, Homi K. Bhabha.-In: Die Beute 14: 48-61.

HALL, S. (1994): Rassismus und kulturelle Identität. Ausgewählte Schriften 2. - Hamburg: Argument.

HaRAWAY, D. (1995): Ein Manifest für Cyborgs. - In: dies. (Hrsg.): Die Neuerfindung der Natur. Primaten, Cyborgs und Frauen. - Frankfurt am Main, New York: 33-72.

Huntington, S. (1993): The Clash of Civilizations? In: Foreign Affair 72, 3: 22-49.

LACoste, Y. (1985): La géographie, ça sert, d'abord, à faire la guerre. -3 . Auflage, Paris: Maspero.

Lossau, J. (2000): Anders denken. Postkolonialismus, Geopolitik und Politische Geographie. - In: Erdkunde 54: 157-168.

NASSEHI, A. (1997): Das stahlharte Gehäuse der Zugehörigkeit. Unschärfen im Diskurs um die «multikulturelle Gesellschaft». - In: ders. (Hrsg.): Nation, Ethnie, Minderheit. - Beiträge zur Aktualität ethnischer Konflikte, Köln, Weimar, Wien: 177-208.

NASSEHI, A. (1998): Die «Welt»-Fremdheit der Globalisierungsdebatte. Ein phänomenologischer Versuch. In: Soziale Welt 49: 151-166.

NaSSeHI, A. (1999): Die Paradoxie der Sichtbarkeit. Für eine epistemologische Verunsicherung der (Kultur-)Soziologie. - In: Soziale Welt 50: 349-362.

Nietzsche, F. (1988): Ueber Wahrheit und Lüge im aussermoralischen Sinne. - In: ders.: Sämtliche Werke. Kritische Studienausgabe. Hrsg. von Giorgio Colli und Mazzino Montinari. Bd. 1. - 2. Auflage, Berlin: 873-890.

Podak, K. (2000): «Gibt es auf Erden ein Maaß?» - Die Zehn Gebote, die aus dem Christentum kommende Moral - und die Schwierigkeiten mit der Ethik nach dem Tod Gottes. - In: Feuilleton-Beilage 
der Süddeutschen Zeitung 94, 22./23./24. April 2000, München: I.

Rajchman, J. (1991): Foucault's art of seeing. - In: ders.: Philosophical events: essays of the 80s, New York: 68-102.

SAID, E. (1978): Orientalism. - New York: Vintage.

SAID, E. (1994): Kultur und Imperialismus. Einbildungskraft und Politik im Zeitalter der Macht. - Frankfurt am Main: Rombach.

SPIVAK, G. C. (1993): Outside in the Teaching Machine. - London, New York: Routledge.

SPYKMAN, N. (1938): Geography and Foreign Policy, II. - In: American Political Science Review 32: 213-236.

StodDART, D. R. (1986): On geography and its history. - Cambridge, Oxford: Blackwell.

WERLEN, B. (1997): Sozialgeographie alltäglicher Regionalisierungen. Bd.2. Globalisierung, Region und Regionalisierung. $-=$ Erdkundliches Wissen 119, Stuttgart: Steiner.

\section{Zusammenfassung: Für eine Verunsicherung des geo- graphischen Blicks: Bemerkungen aus dem Zwischen- Raum}

Die postkoloniale Kritik, die auf einem widersprüchlichen Denken in einem immer wieder anderen Zwischen-Raum basiert, zeichnet sich durch ein entschiedenes Interesse an der De- und Rekonstruktion geographischer Welt-Bilder aus. Dessen ungeachtet hat sich die deutschsprachige Geographie bisher kaum mit den postkolonialen Interventionen auseinandergesetzt. Das ist insofern bedauerlich, als sie viel dazu beitragen können, den geographischen Blick auf die «Ordnung der Dinge» zu bereichern. Vor diesem Hintergrund wird - in Einklang mit den dekonstruktiv-rekonstruktiven Bewegungen des Postkolonialismus und in Auseinandersetzung mit dem derzeit wohl wirkungsmächtigsten Welt-Bild - eine Praxis skizziert, die es erlaubt, immer wieder andere und bessere WeltBilder zu entwerfen. Diese Bilder verzichten ebenso auf den Anspruch, allgemeingültig zu sein, wie sie ohne die fixierende Verortung eines «Eigenen» und «Anderen» auskommen. Damit korrespondieren sie mit einem Denken, das dazu aufruft, gewohnte Denkschemata in Frage zu stellen und nicht zuletzt den eigenen Blick immer wieder aufs neue zu verunsichern.

\section{Summary: Towards a Disturbance of the Geographical Gaze: Remarks from an in-between space}

Operating in an interdisciplinary and inconsistent inbetween space, the critical discourse of postcolonialism shows a decided interest in the deconstruction as well as the reconstruction of geographical imaginations. As such, it can teach valuable lessons to a scientific community which until now has barely paid attention to the nexus of (imperial) power, knowledge, and geography. Against this background and by decon- structing one of the most popular geographical imaginations of the present, a postcolonial and dissident practice of mapping is sketched out. Adopting the perspective of an in-between positionality, this practice dismisses the essentialist categories of the same and the other and, by doing so, leads to other visions, and hopefully improved visions, of cultural localisation. These visions correspond to a way of thinking that appeals for the destabilisation of taken-for-granted truths and, therefore, seeks to disturb the geographical gaze envisioning a geographical reality that supposedly does not argue, but just is.

\section{Résumé: Déstabiliser le regard géographique: remarques relatives à l'espace entre-deux}

La critique postcoloniale, qui se fonde sur une pensée contradictoire dans un entre-les-espaces toujours autre, se caractérise par la volonté de déconstruire et de reconstruire les images géographiques. Toutefois, jusqu'à présent la géographie germanophone n'a guère discuté les interventions postcoloniales. C'est d'autant plus regrettable qu'elles peuvent contribuer à enrichir le regard géographique, qui scrute une réalité prétendant simplement exister sans pouvoir être vue de différentes manières. Tenant compte de ces données, nous esquissons - en prenant pour modèle les mouvements déconstructifs-reconstructifs du postcolonialisme et en discutant l'image actuellement prépondérante - une pratique qui permet d'ébaucher toujours d'autres et de meilleures images géographiques. Celles-ci renoncent autant à une valeur universelle qu'à une localisation fixe d'un «Soi» et d'un «Autre». Ainsi, elles correspondent avec l'appel à remettre en question la manière coutumière de penser et à constamment déstabiliser le propre regard.

Dipl.-Geogr. Julia Lossau, Georgstrasse 16, D-53111

Bonn.

e-Mail: uzs84t@uni-bonn.de 